\title{
Scientific and Technological Resource Sharing Model Based on
} Few-Shot Relational Learning

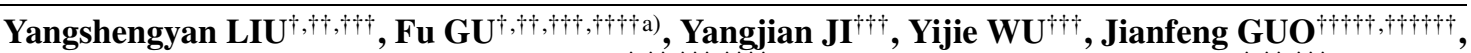

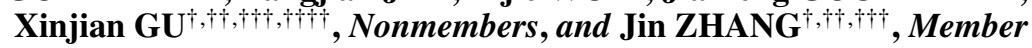

\begin{abstract}
SUMMARY Resource sharing is to ensure required resources available for their demanders. However, due to the lack of proper sharing model, the current sharing rate of the scientific and technological resources is low, impeding technological innovation and value chain development. Here we propose a novel method to share scientific and technological resources by storing resources as nodes and correlations as links to form a complex network. We present a few-shot relational learning model to solve the coldstart and long-tail problems that are induced by newly added resources. Experimentally, using NELL-One and Wiki-One datasets, our one-shot results outperform the baseline framework - metaR by $40.2 \%$ and $4.1 \%$ on MRR in Pre-Train setting. We also show two practical applications, a resource graph and a resource map, to demonstrate how the complex network helps resource sharing.

key words: resource sharing, scientific and technological resource, resource graph, few-shot relational learning
\end{abstract}

\section{Introduction}

Scientific and technological resources are dispensable for both the academic community and industries. However, there lacks of a proper model for resource sharing, resource reuse, and resource integration [1].

The purposes of resource sharing are to improve production, to produce higher quality products, to ensure product safety and transparency, and to reduce the waste of resources [1]. Sharing can improve cluster utilization and avoid data replication and storage waste. Product Lifecycle Management (PLM) [2] is an efficient method for information management throughout the product lifecycle, as it can

Manuscript received November 11, 2020.

Manuscript revised February 20, 2021.

Manuscript publicized April 21, 2021.

${ }^{\dagger}$ The authors are with State Key Laboratory of Fluid Power and Mechatronic Systems, Zhejiang University, Hangzhou, 310027 China.

${ }^{\dagger \dagger}$ The authors are with Key Laboratory of Advanced Manufacturing Technology of Zhejiang Province, Zhejiang University, Hangzhou, 310027 China.

${ }^{\dagger \dagger}$ The authors are with Department of Industrial and System Engineering, Zhejiang University, Hangzhou, 310027 China.

${ }^{t+1 \dagger}$ The authors are with Center of Engineering Management, Polytechnic Institute, Zhejiang University, Hangzhou, 310027 China.

Cttit The author is with School of Public Policy and Management, University of Chinese Academy of Sciences, Beijing, 100049 China.

${ }^{+1+t+\dagger}$ The author is with Institutes of Science and Development, Chinese Academy of Sciences, Beijing 100190, China.

a) E-mail: gufu@zju.edu.cn (Corresponding author)

DOI: 10.1587/transinf.2020BDP0021 tackle heterogeneous resources and track the evolution of these resources. However, resource heterogeneity and enterprise personalization make PLM hard to share resources among different enterprises [2]. Besides, newly arising resources increase at an explosive rate, and their manual annotations cost a lot. Therefore, there is a strong need for a suitable model that can automatically aggregate resources and intelligently share resources based on a handful of labeled materials. The model should also provide resource query and resource sharing to serve the economy. In recent years, knowledge graph (KG) becomes a promising solution for knowledge ordering, as it can serve as a computational model of reality [3]. However, the current literature on knowledge graph mainly focuses on mining semantic knowledge [19] rather than extracting the correlations among scientific and technological resources. Hence, in this sense, the extant knowledge-graph-based approaches might not be applicable for resource ordering and sharing.

In this paper, we aim to construct a resource graph based on few-shot relational learning to effectively integrate various scientific and technological resources in value chains, as well as to share and reuse resources in a more intelligent manner. The resource graph can enhance scientific and technological resource exploitation to increase production efficiency, and it can optimize resource utilization for maximum efficiency and profitability. The resource graph can also achieve several downstream applications such as resource sharing, association query, and graph visualization. Furthermore, to solve the cold-start problem (how to achieve personalized sharing using newly arising resources) and the long-tail problem (distribution imbalance of correlations among resources), we propose a novel few-shot relational learning model. The model combines the meta relation learner with the matching learner to predict the correlations of newly coming resources and to share scientific and technological resources among different task nodes based on the correlations. The experiment results of our method outperform the baseline. A case study of the Chinese home appliance enterprise shows a practical application of resource sharing based on resource graph with few-shot relational learning.

The contributions of our work can be summarized as follows:

1) We develop a resource graph to simplify resource integration and to enhance resource sharing. The 
implementation of resource graph is based on the complex network. It takes the scientific and technological resources as nodes, and the correlations among resources are mapped as links.

2) We propose a new few-shot relational learning model to complete the resource graph. Specifically, our model combines the matching meta with the relation meta to improve the generalization ability of the neighborencoder. The model aims to address the cold-start problem and the long-tail problem, because these problems impede the sharing and utilization of resources.

3) We develop two practical applications, namely a resource graph and a resource map, to effectively facilitate the management and sharing of scientific and technological resources. More downstream applications can be improved using the resource graph, such as intelligent question and answering, semantic querying, and resource reasoning.

The rest of the paper is organized as follows. In Sect. 2, we present and review the current research on the sharing of scientific and technological resources. In Sect.3, our proposed resource graph framework and our few-shot relational learning model are illustrated. Experiments and applications, which demonstrate the performance of our framework, are presented in Sect. 4. Section 5 is reserved for discussion. Finally, Sect. 6 concludes our work and mentions possible future work.

\section{Related Work}

In this section, we present current works on scientific and technological resources. Then we show previous studies on representation learning, as well as several few-shot learning works. Lastly, we list a few significant works on resource sharing. Our work focuses on constructing the complex network for the scientific and technological resources, and the few-shot learning model can intelligently complete the resource graph. The methodology and demonstration will be presented in the next section.

\subsection{Scientific and Technological Resource}

With the development of communities and industries, the information and resources are increased rapidly. It includes the simulation datasets, intellectual resources such as patents and papers, transaction lists, models, and workflows. These resources are highly distributed among schools, companies, and factories, and these scientific and technological resources are significant to the economy [4]. There are works that focused on the scientific and technological resources and we list some of them as follows.

On one hand, there are plenty of works on some special domains using domain-specific resources. For instance, Hong [5] designs a resource management framework for iterative MapReduce processing in large-scale data analysis, but its framework does not tackle knowledge sharing indeed. Kyong [6] provides an optimal resource sharing mechanism in hierarchical VO environments. Takuma [7] analyzes the advantage of distributed representation, and propose a method to extract knowledge with much less annotated data. Ji [8] mines the high-quality reference sentences for joint representation learning of knowledge graphs and text information, and he embeds them to a unified vector space.

On the other hand, there are researchers who not only do research on domain-specific resources, but they study the integration and sharing model of scientific and technological resources. For example, Gu [9] proposes a description model and an establishment method of scientific and technological resources from the perspective of the classification model, metadata model, ontology model, meta-knowledge model, and knowledge graph model. Wang [10] analyzes the related research on scientific and technological resources. $\mathrm{He}$ also finds that although various norms and standards accelerating industrial development appear in the open sharing process, these resources still lack a unified and standardized framework. $\mathrm{Du}$ [11] focuses on human resources using knowledge graph technology. Dong [12] establishes a resource classification system by analyzing the characteristics of different resources.

However, scientific and technological resources lack a proper model for resource classification and integration [13]. We also find that previous studies of knowledge sharing lack the formal basis and do not support knowledge sharing across different systems [13], let alone resource sharing among several enterprises of the industry value chain.

\subsection{Representation Learning and Few-Shot Relational Learning}

With the rapid development of deep learning, representation learning is widely used in multiple research fields such as computer vision, NLP, and knowledge graph [14]. Representation learning aims to transfer the semantic information of the object to a low dimensional vector space where the similarity of two objects is represented by distance.

Structured embedding (SE) [15] is a learning process based on a neural network architecture designed to embed any of these symbolic representations into a more flexible continuous vector space in which the original knowledge is kept and enhanced. Single-layer model[16] improves SE [15] by adding a single layer neural network to learn the nonlinearity of semantic relation. Latent factor model (LFM) [17] is a latent factor model that learns the relationship based on bi-linear transformation. Matrix factorization (RESACL) [18] is a matrix factorization method that directly extracts the vector in low dimension space. Translating based model [19] is the most classic method of representation learning.

However, such embedding-based methods perform poorly where there only have a few associative triples in training. This problem in the knowledge graph is usually 
called the few-shot learning problem. FewRel [20] is a classic Few-Shot Relation Classification Dataset that includes 70,000 sentences on 100 relations derived from Wikipedia and annotated by crowd workers. Xiong [21] propose a oneshot relational learning framework that utilizes the knowledge extracted by embedding models, and learns a matching metric by considering both the learned embeddings and one-hop graph structures. Chen [22] focuses on transferring relation-specific meta information to make the model learn the most important knowledge and learn faster. FSRL [23] is a novel few-shot relation learning model that aims at discovering facts of new relations with few-shot references. MLMAN [24] presents a multi-level matching and aggregation network for the task of classifying few-shot relation.

The previous studies mostly focus on extracting semantic knowledge from texts [19], [25] rather than identifying the correlations among scientific and technological resources. This problem seriously impedes resource sharing. Besides, the continuously arising resources of the industry value chain also hinder scientific and technological resource sharing.

\subsection{Resource Sharing}

Resources of industry value chain can be classified into scientific and technological resources and business resources. Scientific and technological resources include patent, paper, conference, standard, and other academic resources. Business resources represent a variety of data from production to marketing.

Resource sharing plays an important role in knowledge management. Specifically, transferring and disseminating resources from one task node to another can reduce costs and promote productivity. Sato [26] develops a knowledgesharing system using XML linking language and peer-topeer technology to facilitate intellectual creative work and knowledge management. Zhang [27] focuses on how can individuals and groups share knowledge on the Internet more efficiently, and the knowledge resources in the network are redefined by object-oriented thinking. They finally propose a three-layer knowledge-sharing model. Semantic web technology is another available framework for resource sharing [28]. Raza [29] adds a knowledge layer to commercial PLM systems to solve the semantic interoperability problem of heterogeneous data. Arduin [30] proposes semantic interoperability between a collaborative platform and a product lifecycle management (PLM) system to share knowledge within extended enterprises. He also highlights the conditions under which a piece of information shared through a PLM system may lead to one and only one interpretation. Assouroko [31] proposes a semantic relationship management approach to improve knowledge management and reuse in collaborative product development.

Nowadays, knowledge graph with representation learning has become more prevalent to extract knowledge [32], [33]. We find it can be a more intelligent method to improve resource sharing significantly. However, we realize that few works take full advantage of this technique to share scientific and technological resources. In the next section, we describe the scientific and technological resource graph for resource sharing in detail. We also introduce our few-shot relational learning model to figure out the cold-start problem and the long-tail problem when encountering newly added resources.

\section{Method}

\subsection{Resource Sharing Based on Resource Graph}

Scientific and technological resources is one of the primary productive forces for developed and developing countries. However, scientific and technological resource sharing and allocation rates are still low, and the current situation of resource sharing cannot meet production status [34]. The barrier of resource sharing caused by resource disperse and resource islands also becomes more severe. Thus, resource dissemination among urban agglomerations is deficient. These problems restrict technological innovation and the harmonious development of urban agglomerations.

Therefore, it is necessary to design a model for scientific and technological resource sharing and allocation. We develop a resource graph by storing resources as nodes and discovering correlations as links to form a complex network for resource integration and resource sharing. Further, we propose a few-shot relational learning model to predict correlations among resources based on the links. Thus, resources can be shared among task nodes of the different stages of the value chain.

Resource sharing can be described as a process either of push or pull [35]. Resource push is to push resources like newsletters or subscriptions to the users, and resource pull represents the activity of consumer seeking out resources such as library search, seeking out an expert, collaborating with a high-tech enterprise. Resource sharing can be further divided into explicit resource sharing and tacit resource sharing. Explicit resource sharing [35] is impacted by the factors such as articulation of users, the awareness of resource availability and access, the guidance of the sharing system, and resource completeness. Contrarily tacit resource sharing [35], such as informal networks among human resources, has its relevant factor that is hard to identify and requires the model for employees to foster informal networks and trade tacit knowledge. Generally, it is much more important for the sharing model to externalize the source of tacit knowledge and pass on the resource in practice. Broadly speaking, the resource sharing model must implement the right processes and frameworks, and it can foster a resource sharing culture [34].

Specifically, we divide resources into scientific and technological resources and business resources. The former represents research on the social and natural world through observation and experiment. The latter consists of data resources involved in the process of production, marketing, and after-sale service. Business resources should also 


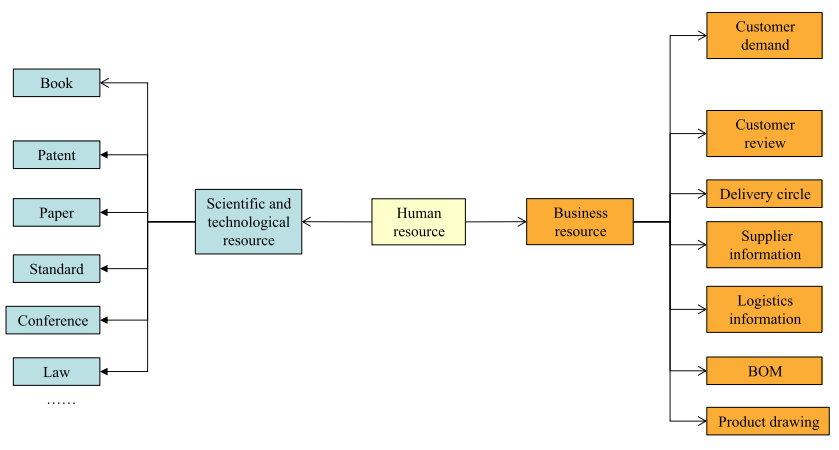

Fig. 1 Resource graph schema.

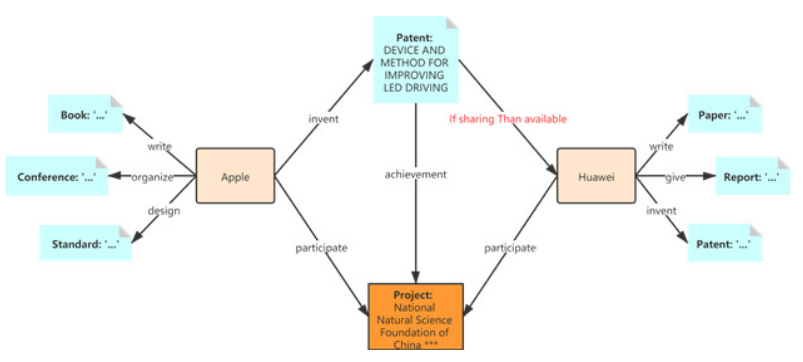

Fig. 2 Resource sharing instance based on resource graph.

contain tacit knowledge like experience and reference [35]. Then a resource graph can be constructed using the scientific and technological resources. Each node in the graph represents an individual resource instance, and the correlations among resources are set as links. It is different from the general knowledge graph in which a node may be a noun or phrase of a specific description. A resource graph is set up for the urban agglomerations or the industry to integrate various resources, and it provides a proper model for resource sharing. The proposed resource graph can also support administrators to make decisions and visualize resource allocation easily.

As shown in Fig. 1, a resource graph takes human resources as the graph center, which can support resource sharing based on the correlations among people. Noticeably, the human resource in Fig. 1 is regarded as a symbol for enterprises or urban agglomerations, and the schema is regarded as the classification tree to some extent.

As shown in Fig. 2, a node in the resource graph corresponds to a specific resource, and resources around a specific human resource node are in a resource pool that supports resource sharing. Resource sharing is based on the resource graph hugely, and once constructed; one can share resources to another according to rules like "If... Than..." (marked in red in Fig. 2). The main purpose is to offer more scientific and rational decision support and to share resources to drive the development and integration of the regional economy.

\subsection{Few-Shot Relational Learning}

The resource graph is already available to achieve resource integration and resource sharing as shown in Fig. 2. However, we find that it still has many problems limiting the ability of resource sharing in practice as bellow.

On one hand, the cold-start problem is common in recommendation systems [36]. It also impacts the resource sharing performance indeed. We find that this problem stems from three factors: new scientific and technological resources, new business resources, and new human resources [37]. These newly arising resources appear continuously with the development of industry. But they are unlabeled and costly for manual annotation. Thus, it is hard to add new resources to the resource graph, let alone resource sharing based on links.

On the other hand, we discover that the resource graph built for urban agglomerations and enterprises will encounter the long-tail problem [38]. Because those newly added relations often do not have enough triples, and it will be ignored as time goes by [39]. This problem will lead to resource waste and incompleteness of the resource graph. Besides, resource sharing will be much more biased because the model just focuses on the resources that have a large number of relations around human resources.

Therefore, we regard these problems as the few-shot learning problem, and we propose a few-shot relational learning model to complete the resource graph. Few-shot relational learning aims at predicting new facts under a challenging setting where only a few known instances are available [21]. The previous study also finds that only observing a few associative triples is enough to achieve the few-shot link prediction [22]. Thus, our method aims to complete the resource graph via newly arising resources and to predict relations or tail resources for users to support scientific and technological resource sharing. Namely, the model tries to achieve intelligence in the aggregation, sharing, and utilization of scientific and technological resources to serve the real economy.

In detail, our method is inspired by two previous work [21], [22], and we try to improve the performance by combining their main contributions, as shown in Fig. 3. Xiong [21] proposes a one-shot relational learning framework that learns a matching metric to imitate the existing similar facts. Chen [22] tries to learn several relationspecific meta and transfer them to newly unknown triples. However, Xiong [21] ignore the circumstance that there may exist unseen relations, and it cannot merely learn by a simple matching matric. It is common for the resource graph because the newly coming resources may become unpredictable. On the other hand, however, the one-hop graph structures dose not fully utilized by metaR [22]. It loses the structure information around human resources and hinders resource sharing.

As shown in Fig. 4, our method aims to aggregate newly added resources into the corresponding one-hop graph, which could effectively the robustness and generalization of the whole model. We use meta relation learner to fit new relations not included in the existing graph structure. Then we aggregate all one-hop structure information 


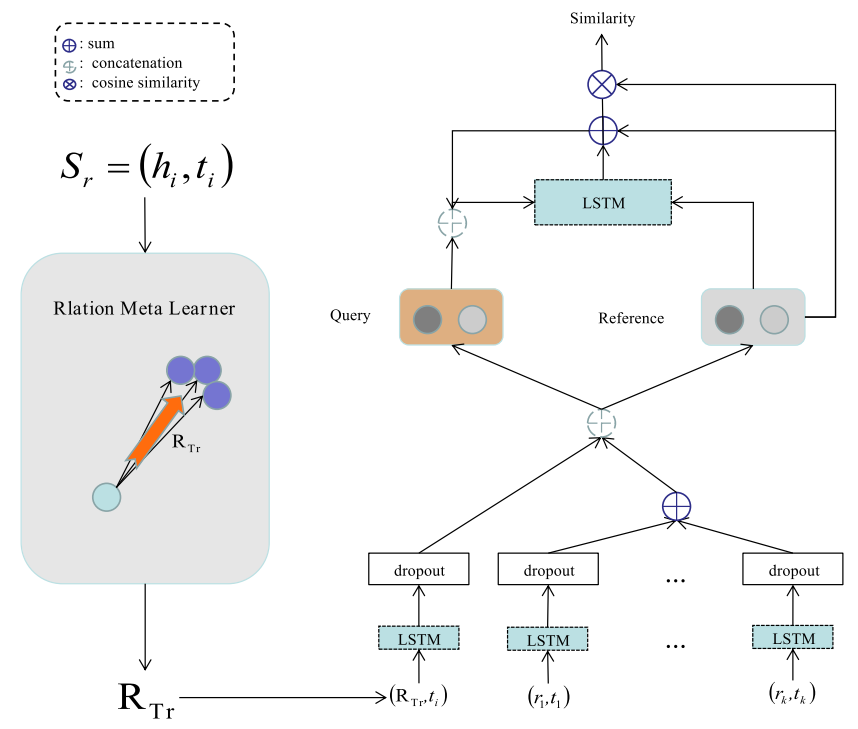

Fig. 3 The model of few-shot relational learning.

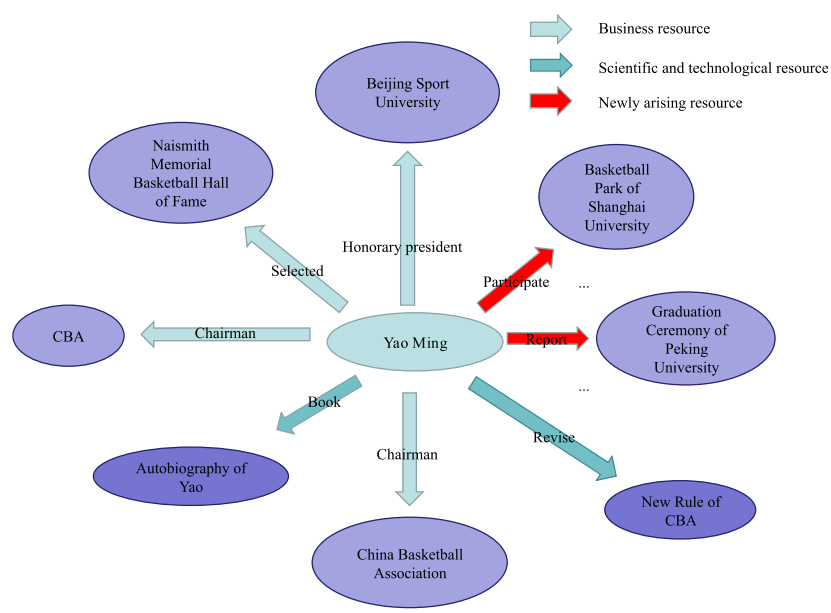

Fig. 4 Aggregating newly arising resources into one-hop graph.

with the aforementioned meta relation to match the query. We believe our method can adapt to any new resources and complete the resource graph for downstream resource sharing task.

We first embed resources into vector space using pretrained embeddings such as Glove [40] or BERT [41]. We add all the descriptions and attributes of a single resource as the descriptor to represent itself because we firmly believe that the semantic information inside the resource node is helpful to multiple downstream tasks. Then we use the same idea as Chen [22] to construct the meta relation learner. The single difference is we further add an LSTM layer to capture the sequential information in the input layer as Eq. (1):

$$
\begin{aligned}
& \mathbf{x}_{\mathrm{sp}}^{0}=\operatorname{LSTM}\left(h_{i} \oplus t_{i}\right) \\
& \mathbf{x}_{\mathrm{sn}}^{0}=\operatorname{LSTM}\left(h_{i} \oplus t_{j}\right)
\end{aligned}
$$

where $h_{i}$ and $t_{i}$ are pre-trained embeddings of the head entity and tail entity separately. $\oplus$ represents the concatenation operation.

Then the meta relation for the input fact pair is calculate via an $L$-layers stacked LSTM module as follows:

$$
\begin{aligned}
& \mathbf{x}^{l}=\operatorname{LSTM}\left(\mathbf{x}^{l-1}\right) \\
& \mathbf{R}_{\left(h_{i}, t_{i}\right)}=\operatorname{LSTM}\left(\mathbf{x}^{L-1}\right) \\
& \mathbf{R}_{\mathrm{Tr}}=\frac{\sum_{i=1}^{K} \mathbf{R}_{\left(h_{i}, t_{i}\right)}}{K}
\end{aligned}
$$

where $l \in\{1, \ldots, L-1\}$ represents the $l_{\text {th }}$ layer of the stacked LSTM module. $\mathbf{x}^{l}$ is the embedding of the $l_{\text {th }}$ layer. $\mathbf{R}_{\left(h_{i}, t_{i}\right)}$ is the specific meta relation of input $h_{i}, t_{i}$. $\mathbf{R}_{\mathrm{Tr}}$ represents the total meta relation of the selected task in every learning epoch. $K$ is the number of facts.

We calculate the score of each fact in Eq. (4). The score function is similar to TransE [19].

$$
S_{1}\left(h_{i}, t_{i}\right)=\left\|h_{i}+\mathbf{R}_{\mathrm{Tr}}-t_{i}\right\|_{2}^{2}
$$

Then we do a similar process as Xiong [21] called neighbor encoder to grab one-hop structure around the resource node. Function $f()$ means to learn the characteristic of adjacency. The difference in Eq. (5) is that we add the external pair of meta relation into the candidate pairs where $\mathrm{R}_{\mathrm{Tr}}$ and $t_{k}$ are features of the meta relation and features of the tail resources, and $\mathbf{X}_{r_{k}, t_{k}}=\operatorname{LSTM}\left(r_{k} \oplus t_{k}\right)$.

$$
f\left(N_{r}\right)=\sigma\left(\frac{1}{\left|N_{r}\right|} \sum_{\left(r_{k}, t_{k}\right),\left(\mathrm{R}_{\mathrm{Tr}}, t_{k}\right) \in N_{r}} \mathbf{X}_{r_{k}, t_{k}}\right)
$$

We do the same matching processor based on cosine similarity just the same as Xiong [21] in Eq. (6) where $s=$ $f\left(N_{h_{0}}\right) \oplus f\left(N_{t_{0}}\right), q=f\left(N_{h_{i}}\right) \oplus f\left(N_{t_{i j}}\right)$, and $k$ is a hyperparameter to be tuned.

$$
\begin{aligned}
& h_{k+1}^{\prime}, \mathbf{X}_{r_{k}, t_{k}}=\operatorname{LSTM}\left(q,\left[h_{k} \oplus s, \mathbf{X}_{r_{k}, t_{k}}\right]\right) \\
& h_{k+1}=h_{k+1}^{\prime}+q \\
& S_{2}^{k+1}=\frac{h_{k+1} \cdot s}{\left\|h_{k+1}\right\|\|s\|}
\end{aligned}
$$

Lastly, the total score $s_{\text {total }}$ is the sum of two scores in formula 4 and formula 6 above. $s_{\text {total }}$ and $s_{\text {total }}$ are the scores of the positive fact and the score of the negative one. Then we conduct margin ranking loss $L\left(S_{\gamma}\right)$ based on the total score of input fact as Eq. (7) where $\gamma$ is another hyperparameter to be tuned.

$$
\begin{aligned}
& s_{\text {total }}=S_{1}\left(h_{i}, t_{i}\right)+S_{2}^{k+1} \\
& L\left(S_{r}\right)=\sum_{\left(h_{i}, t_{i}\right) \in S_{r}}\left[\gamma+s_{\text {total }}^{+}-s_{\text {total }}^{-}\right]_{+}
\end{aligned}
$$

Notice that the meta relation fed into the matching network represents the idea that not all the relationships are already involved in the matching network, especially for newly arising resources. Meanwhile, the local one-hot graph structure embeds more semantic information to learn the meta relation. Our method brings a lot from these two previous works. We actually combine their advantages and utilize 
our method in resource graph to learn the latent relationships for resource sharing.

\section{Experiment}

Our few-shot relational learning model is tested on two public datasets NELL-One and Wiki-One selected by Xiong [21] from NELL and Wiki data. We also show a resource sharing case of a home appliance enterprise based on our method. We further discuss and analyze the experiment results in the end.

\subsection{Datasets and Evaluation Metrics}

We use two few-shot learning datasets as previous studies [21], [22] for a better comparison of performance on these two benchmarks. We also use the same evaluation metrics for convenience.

Datasets: We first experiment on two public datasets, NELL-One and Wiki-One, the same as Chen [22]. These datasets are first proposed by Xiong [21], and they are further used by Chen [22]. NELL-One and Wiki-One are used for few-shot relation learning. Namely, given the fact (the Arlanda Airport, Stockholm), the algorithm needs to infer the fact (the Haneda Airport, Tokyo). We divide these datasets into train sets, validation sets, and test sets. The statistics are shown in Table 1 where Fit in Table 1 means neither Pre-Train setting [22] nor In-Train setting [22].

Evaluation Metrics: We use the same evaluation methods as Xiong [22], including MRR and Hits@N. MRR represents the mean reciprocal rank, and Hits@N means the proportion of correct predictions ranked in the top $\mathrm{N}$ in link prediction tasks.

\subsection{Implementation}

We use Adam optimizer and ReLU activation function to achieve better convergence in training. The initial learning rate is 0.01 , and it descends to 0.0001 gradually to reach the global optima of the whole training model with fast convergence [22]. The embedding size, following the work of Xiong [21], are 100 and 50 for NELL-One and Wiki-One respectively. We use the early stop trick when the model hits the best performance on Hits@10. To get better performance, the hidden size and the layer of LSTM are 200 and 4 for NELL-One, and 100 and 4 for Wiki-One.

\subsection{Result}

\subsubsection{Experiment Results}

To compare with state of the art, we experiment on two kinds of few-shot link prediction tasks, 1-shot and 5-shot, on both datasets.

As shown in Table 2, our method is better than the baseline in any setting. On one hand, compared to MetaR [22] with the Pre-Train setting, we increase 1-shot results of NELL-One by $40.2 \%, 6 \%, 21 \%$, and $73 \%$ on MRR, Hits@10, Hits@5, Hits@1, and 5-shot results by 14.8\%,6.5\%,16.1\% and 17\% on MRR, Hits@ 10, Hits@5, Hits@1. Besides, we increase 1-shot results of Wiki-One by 4.1\%,4.2\%, 1.1\%, and 4.1\% on MRR, Hits@ 10, Hits@5, Hits@1, and 5-shot results by 3.7\%,6.5\%, 4.2\% and 2.6\% on MRR, Hits@10, Hits@5, Hits@1. On the other hand, compared with the In-Train setting, we increase 1-shot results of NELL-One by $24 \%, 19.2 \%, 22 \%$, and $28.2 \%$ on MRR, Hits@10, Hits@5, Hits@1, and 5-shot results by 28.4\%, 17.4\%,22\% and 33.3\% on MRR, Hits@ 10, Hits@5, Hits@1. Meanwhile, we increase 1-shot results of WikiOne by $4.1 \%, 3.9 \%, 5.6 \%$, and 1.9\% on MRR, Hits@10, Hits@5, Hits@1, and 5-shot results by 4.5\%,13.6\%,6.8\%, and 5.6\% on MRR, Hits@10, Hits@5, Hits@1.

We can conclude that the performance of few-shot link prediction is affected by the quality and quantity of the data indeed. On one hand, the Pre-Train setting is better than the In-Train setting under large datasets, such as Wiki-One. That is because of the sparsity problem of the entities. It is difficult to learn the correct meta relation when the support triples are too sparse, which is unstable and biased for the meta-based relation learner. That is why pre-trained embeddings improve 1-shot tasks a lot. On the other hand, the performance improved under the In-Train setting for the small dataset NELL-One, because fitting background brings more sparsity into training procedure.

\subsubsection{Ablation Study}

We have proved that the information in meta relation successfully enhances the ability of the neighbor encoder [21]. To further figure out the significance of correlations, we conduct an ablation study on NELL-One with metric Hit@10 with seven settings. The results are shown in Table 3. The first one is removing both the meta relation learner and the GMatching module, which simplifies the model to a classical TransE embedding model, denoted as $-m g-n g-r$. The second one is removing the meta relation learner, which becomes the GMatching_TransE, denoted as $-r$. The third one is removing both the matching processor and the neighbor encoder, denoted as $-m g-n g$. The fourth one is removing the matching processor, denoted as $-m g$. The fifth one is removing the neighbor encoder, denoted as $-n g$. The sixth one is the standard metaR. The last one is our complete model denoted as standard. Specifically, the results of both the 
Table 2 Experiment results of few-shot relational learning.

\begin{tabular}{|c|c|c|c|c|c|c|c|c|c|}
\hline \multirow{2}{*}{\multicolumn{2}{|c|}{ NELL-One }} & \multicolumn{2}{|c|}{ MRR } & \multicolumn{2}{|c|}{ Hits@10 } & \multicolumn{2}{|c|}{ Hits@5 } & \multicolumn{2}{|c|}{ Hits@1 } \\
\hline & & 1-shot & 5-shot & 1-shot & 5-shot & 1 -shot & 5-shot & 1-shot & 5-shot \\
\hline \multicolumn{2}{|l|}{ GMatching_RESCAL } & .188 & - & .305 & - & .243 & - & .133 & - \\
\hline \multicolumn{2}{|l|}{ GMatching_TransE } & .171 & - & .255 & - & .210 & - & .122 & - \\
\hline \multicolumn{2}{|l|}{ GMatching_DisMult } & .171 & - & .301 & - & .221 & - & .114 & - \\
\hline \multicolumn{2}{|l|}{ GMatching_ComplEx } & .185 & .201 & .313 & .311 & .260 & .264 & .119 & .143 \\
\hline \multicolumn{2}{|l|}{ GMatching_Random } & .151 & - & .252 & - & .186 & - & .103 & - \\
\hline \multirow{2}{*}{ MetaR } & Pre-Train & .164 & .209 & .331 & .355 & .238 & .280 & .093 & .141 \\
\hline & In-Train & .250 & .261 & .401 & .437 & .336 & .350 & .170 & .168 \\
\hline \multirow{2}{*}{ Our method } & Pre-Train & .230 & .240 & .351 & .378 & .288 & .325 & .161 & .165 \\
\hline & In-Train & .310 & .335 & .478 & .513 & .410 & .427 & .218 & .224 \\
\hline \multicolumn{2}{|c|}{ Wiki-One } & 1-shot & 5-shot & 1-shot & 5-shot & 1-shot & 5-shot & 1-shot & 5-shot \\
\hline \multicolumn{2}{|c|}{ GMatching_RESCAL } & .139 & - & .305 & - & .228 & - & .061 & - \\
\hline \multicolumn{2}{|l|}{ GMatching_TransE } & .219 & - & .328 & - & .269 & - & .163 & - \\
\hline \multicolumn{2}{|l|}{ GMatching_DisMult } & .222 & - & .340 & - & .271 & - & .164 & - \\
\hline \multicolumn{2}{|l|}{ GMatching_ComplEx } & .200 & - & .336 & - & .272 & - & .120 & - \\
\hline \multicolumn{2}{|l|}{ GMatching_Random } & .198 & - & .299 & - & .260 & - & .133 & - \\
\hline \multirow{2}{*}{ MetaR } & Pre-Train & .314 & .323 & .404 & .418 & .375 & .385 & .266 & .270 \\
\hline & In-Train & .193 & .221 & .280 & .302 & .233 & .264 & .155 & .178 \\
\hline \multirow{2}{*}{ Our method } & Pre-Train & .327 & .335 & .421 & .445 & .379 & .401 & .277 & .277 \\
\hline & In-Train & .201 & .231 & .291 & .343 & .246 & .282 & .158 & .188 \\
\hline
\end{tabular}

Table 3 Results of ablation study on Hits@ 10 of 1-shot link prediction in NELL-One.

\begin{tabular}{lcc}
\hline Ablation Conf. & 1-shot(Pre-Train/In-Train) & 5-shot(Pre-Train/In-Train) \\
\hline$-m g-n g-r$ & $.192 / .192$ & - \\
$-r$ & $.255 / .255$ & - \\
$-m g-n g$ & $.339 / .415$ & $.359 / .447$ \\
$-m g$ & $.343 / .422$ & $.362 / .463$ \\
$-n g$ & $.349 / .453$ & $.367 / .491$ \\
metaR & $.331 / .401$ & $.355 / .437$ \\
standard & $.351 / .478$ & $.378 / .513$ \\
\hline
\end{tabular}

first setting and the second setting are neither Pre-train nor In-train, and they are copied from Xiong [21].

On one hand, Table 3 shows that, compared to the standard results of the 1-shot setting, removing the matching processor decreases $2.3 \%$ and $11.7 \%$ on two dataset settings, and removing the neighbor encoder decreases $0.6 \%$ and $5.2 \%$. Removing both the matching processor and the neighbor encoder continuously decreases the performance by $3.4 \%$ and $13.2 \%$. On the other hand, when the shot number arising to 5 , the performance increases by $7.7 \%$ and $7.3 \%$ under the standard setting, and it has the same upward trend under every setting. Thus, both the meta relation learner and the GMatching module contribute to the fewshot link prediction task dramatically. Moreover, more shot samples provide better performance because these training samples make the meta relation more robust and powerful. It also illustrates that the learned meta relation completes the information of neighbor encoder, which is significant and helpful for the few-shot link prediction.

\subsubsection{Shot Number Analysis}

As our model combines the neighbor-encoder with the meta relation, here we try to analyze how these relations affect

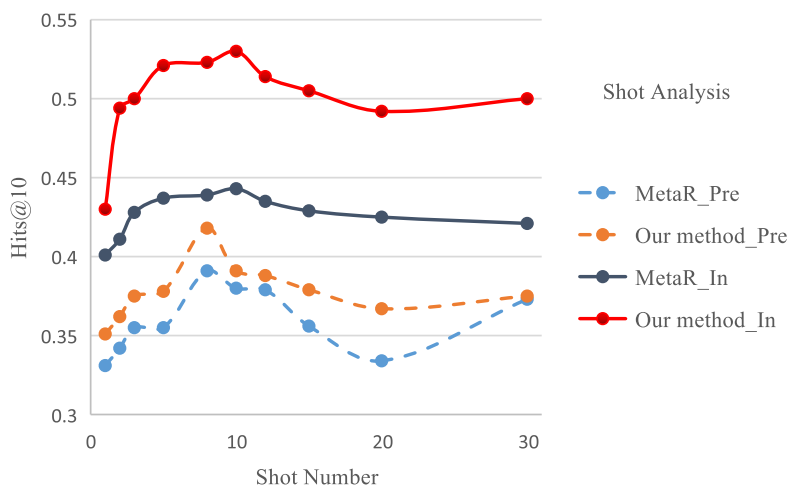

Fig. 5 Shot number analysis.

the model convergence. Figure 5 shows the learning curves of different shot numbers. We evaluate these curves on the Hits@10 on the validation set.

Obviously, we find that more shot samples generally lead to better performance of the proportion of correct link predictions (Hits@10). The result is similar to the analysis [21] on neighbor-encoder. Because both increasing the neighbors and adding more shot samples enrich the information of the fact. We also observe that 10 shots for each task is enough to learn a robust meta relation, and more samples seem to be saturated. The potential reason is that more samples for training can reduce the over-fitting of the meta-learning model. But it also brings noises to the model. In this situation, fine-tuning models could be better alternatives.

\subsection{Application}

We show two practical applications of resource sharing based on the resource graph with few-shot relational learn- 


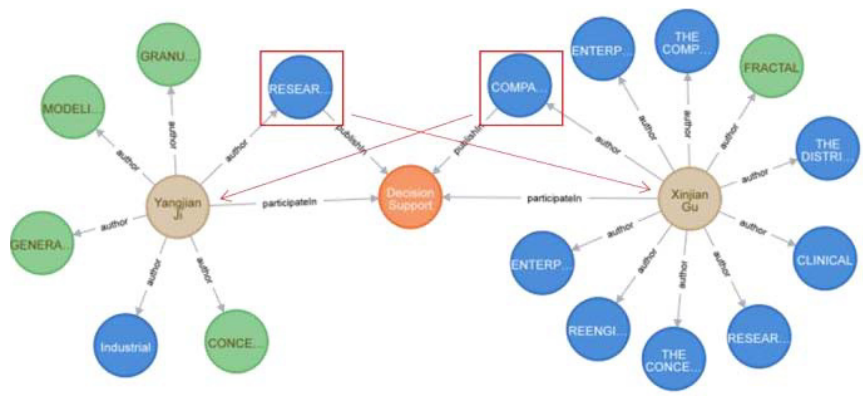

(a)

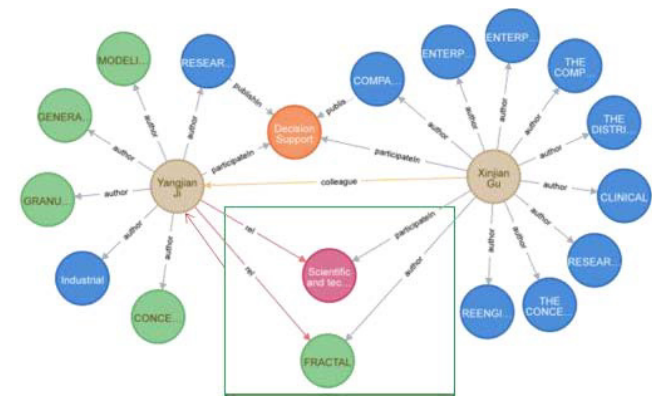

(b)

Fig. 6 Instance of resource sharing using our method.

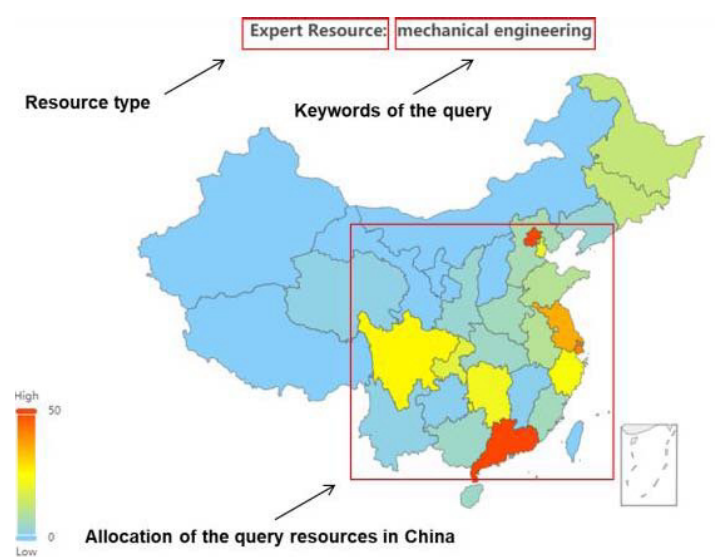

(a)

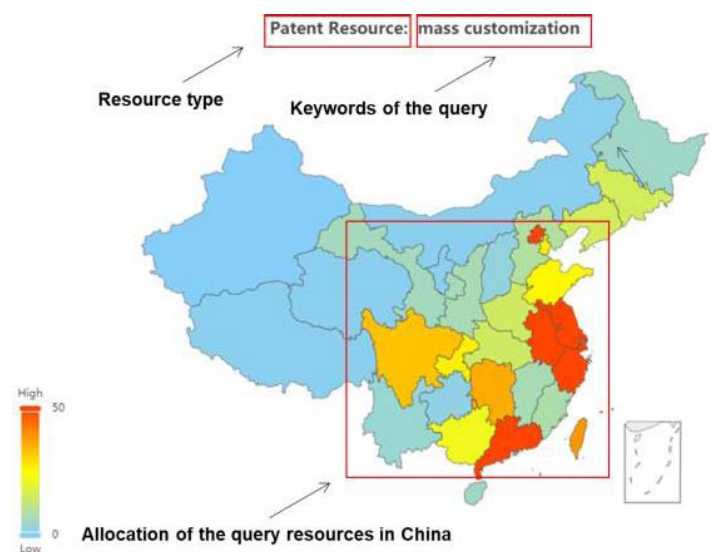

(b)

Fig. 7 Resource map based on resource graph.

ing. We construct a resource service platform for a Chinese home appliance enterprise. The platform relies on a specific resource graph to link various scientific and technological resources. It also provides the interface to share resources among different task nodes.

As shown in Fig. 6 (a) and Fig. 6 (b), our method helps the experts share scientific and technological resources (marked in the red square) with each other by the correlations among resource nodes because they both attend the same conference and publish their papers. The predictive relations based on our few-shot relational learning model also provide the support of new resource sharing (marked in the green square). As shown in Fig. 6(b), expert Gu may share the patent resource and the project resource to expert Ji based on the predictive correlations (marked as "rel"). The visualization of the resource graph supports its executors to reach a swift decision, and resource sharing could be friendly by clicking the resource node and choose the target task node easily.

Figure 7 shows the resource map that depicts the resource distribution of urban agglomeration. The map supports the semantic query of resources such as expert, high-tech enterprise, agency, patent, and other scientific and technological resources. Figure 7 (a) shows the scientific and technological resource allocation of specific query input, and Fig.7 (b) is the corresponding information of agency in the selected urban agglomeration. Obviously, it is effective and convenient to share scientific and technological resources via the resource map.

\section{Analysis and Discussion}

Most relevant resource sharing models merely classify and store their data in a closed domain such as product data management (PDM) and manufacturing execution system (MES), which hugely limits the horizontal sharing across different systems. However, other downstream tasks based on knowledge graphs ignore the practical applications of scientific and technological resource integration and sharing. As far as we know, we are the first to try to achieve scientific and technological resource ordering and resource sharing based on knowledge graph technology. We actually improve it to construct a novel resource graph that can be a more suitable business model for the integration and sharing of scientific and technological resources. Furthermore, at present, most researchers developed recommendation systems to share resources, but such systems are hard to solve the cold-start problem caused by new resources. Therefore, our few-shot relational learning model could be a proper solution to some extent.

Specifically, we emphasize that a normalized resource classification tree defining in Sect.3.1 as resource graph 
Table 4 Abbreviation.

\begin{tabular}{|c|c|}
\hline \multicolumn{2}{|l|}{ Abbreviation } \\
\hline PLM & Product Lifecycle Management \\
\hline PDM & Product Data Management \\
\hline MES & Manufacturing Execution System \\
\hline KG & Knowledge Graph \\
\hline long-tail proble & $\begin{array}{l}\text { How to achieve personalized sharing using newly arising } \\
\text { resources }\end{array}$ \\
\hline $\begin{array}{l}\text { cold-start } \\
\text { problem }\end{array}$ & Distribution imbalance of correlations among resources \\
\hline $\mathrm{SE}[15]$ & A structured embedding method \\
\hline LFM[17] & A latent factor model \\
\hline FewRel[20] & A classic Few-Shot Relation Classification Dataset \\
\hline FSRL[23] & A novel few-shot relation learning model \\
\hline MLMAN[24] & A multi-level matching and aggregation network \\
\hline
\end{tabular}

schema is the basis of resource graph construction. Then a resource graph can be efficiently constructed based on the explicit correlations among resources efficiently. Furthermore, a few-shot relational learning model is used to learn a general meta-model to predict implicit links among new resources and complete the resource graph via predictable correlations. Our model practically combines the characteristic of two previous works based on the intuitive that both the structure information and the implicit information deep inside meta relations are important for the few-shot link prediction. Finally, a more robust and completive resource graph can effectively support users to share and manage scientific and technological resources.

However, the limitations of our method are also obvious. On one hand, the construction of the resource graph is hugely influenced by the specialists using top-down methods. And the performance of bottom-up methods in most realistic scenarios, such as unsupervised learning, are usually unsatisfactory. On the other hand, the few-shot relational learning model is supposed to complete the resource graph automatically. However, the performance of our meta-learning model is still have significant room for improvement. Thus, more attention is required to refine and optimize such deep learning models to achieve real cognitive intelligence.

We list the abbreviations of the whole paper in Table 4.

\section{Conclusion and Future Work}

In this paper, we propose a scientific and technological resource sharing method based on the resource graph. We also present a novel few-shot relational learning model for the few-shot link prediction to solve the long-tail problem and cold-start problem, which aims to share newly arising resources and complete resource graph intelligently. Lastly, the experiments on the two public datasets have proved the reliability and efficiency of our proposed method. We also show the practical examples of our resource sharing and query based on the corresponding resource graph.

In the future, we may include and modify more metalearning methods, such as reinforcement learning and generative adversarial learning, to further improve the perfor- mance of few-shot relation learning. We also tend to develop more applications based on the resource graph to facilitate urban agglomerations and industries.

\section{Acknowledgments}

This research is funded by National Key R\&D Program of China (no. 2017YFB1400302), National Natural Science Foundation of China (nos. $71901194 \&$ \& 51775493), and Science and Technology Innovation 2025 Major Project of Ningbo (No. 2018B10069).

\section{References}

[1] Y. Nie, "Study on Relationship between Scientific and Technological Resource Sharing and Regional Economic Development," Proc. 2016 International Conference on Education, Sports, Arts and Management Engineering, Atlantis Press, pp.241-245, 2016.

[2] A.P. Emmanuel, D.J. Le, A.M. Hélène, et al., "Knowledge sharing within extended enterprises: Case of product lifecycle management systems," European Conference on Knowledge Management, Portugal, pp.63-71, 2014.

[3] Y. Cao, Z. Hu, T. Chua, Z. Liu, and H. Ji, "Low-Resource Name Tagging Learned with Weakly Labeled Data," The Conference on Empirical Methods in Natural Language Processing (EMNLP 2019), pp.261-270, 2019.

[4] J. Guo, J. Pan, J. Guo, F. Gu, and J. Kuusisto, "Measurement framework for assessing disruptive innovations," Technological Forecasting and Social Change, vol.139, pp.250-265, 2019.

[5] S. Hong, K. Park, C.-D. Lim, and J.-W. Chang, "Cancelled: A New Efficient Resource Management Framework for Iterative MapReduce Processing in Large-Scale Data Analysis," IEICE Trans. Inf. \& Syst., vol.E100-D, no.4, pp.704-717, 2017.

[6] K.H. Kim, G.M. Tchagoue, T.-K. Jun, and W.Y. Lee, "An Optimal Resource Sharing in Hierarchical Virtual Organizations in the Grid," IEICE Trans. Inf. \& Syst., vol.E95-D, no.12, pp.2948-2951, 2012.

[7] T. Ebisu and R. Ichise, "Triple Prediction from Texts by Using Distributed Representations of Words," IEICE Trans. Inf. \& Syst., vol.E100-D, no.12, pp.3001-3009, 2017.

[8] Z. Ji, Z. Lei, T. Shen, and J. Zhang, "Joint Representations of Knowledge Graphs and Textual Information via Reference Sentences," IEICE Trans. Inf. \& Syst., vol.E103-D, no.6, pp.1362-1370, 2020.

[9] F. Gu, Y. Liu, and X. Gu, "Description method of scientific and technological resources," Knowledge Management Forum, vol.5, no.2, 2020.

[10] Z. Wang and Q. Yang, "Research on the standard system of open sharing of scientific and technological resources," China Information Review, vol.48, no.4, pp.19-23, 2016.

[11] P. Du, T. Wu, and C. Wang, "Analysis of knowledge map in the field of human resource research in science and technology," Forum on science and technology in China, vol.8, pp.83-89, 2013.

[12] M. Dong, Y. Sun, and B. Wang, "Research on scientific and technological resources and its classification system," Co-operative economy \& science, vol.10, pp.28-30, 2014.

[13] T. Gu, X. Wang, and D. Zhang, "An ontology-based context model in intelligent environments," arXiv preprint arXiv: 2003.05055, 2020.

[14] Z. Liu, M. Sun, Y. Liu, et al., "Knowledge representation learning: A review," Journal of Computer Research and Development, vol.53, no.2, pp.247-261, 2016.

[15] A. Bordes, J. Weston, R. Collobert, et al., "Learning structured embeddings of knowledge bases," AAAI Conference on Artificial Intelligence, DBLP, pp.301-306, 2011.

[16] A. Bordes, X. Glorot, J. Weston, and Y. Bengio, "A semantic matching energy function for learning with multi-relational data," Machine Learning, vol.94, no.2, pp.233-259, 2014. 
[17] R. Jenatton, N.L. Roux, A. Bordes, et al., "A latent factor model for highly multi-relational data," International Conference on Neural Information Processing Systems, pp.3167-3175, 2012.

[18] N. Maximilian, V. Tresp, and H.-P. Kriegel, "Factorizing YAGO: Scalable Machine Learning for Linked Data," Annual Conference on World Wide Web, pp.271-280, 2012.

[19] B. Antoine, U. Nicolas, G. Alberto, et al., "Translating embeddings for modeling multi-relational data," Neural information processing systems, 2787-2795, 2013.

[20] X. Han, H. Zhu, P. Yu, Z. Wang, Y. Yao, Z. Liu, and M. Sun, "Fewrel: A large-scale supervised few-shot relation classification dataset with state-of-the-art evaluation," Proc. 2018 Conference on Empirical Methods in Natural Language Processing (EMNLP), pp.4803-4809, 2018.

[21] W. Xiong, M. Yu, S. Chang, X. Guo, and W.Y. Wang, "One-shot relational learning for knowledge graphs," Proc. 2018 Conference on Empirical Methods in Natural Language Processing (EMNLP), pp.1980-1990, 2018.

[22] M. Chen, W. Zhang, W. Zhang, et al., "Meta relational learning for few-shot link prediction in knowledge graphs," Proc. 2019 Conference on Empirical Methods in Natural Language Processing (EMNLP), pp.4208-4217, 2019.

[23] C. Zhang, H. Yao, C. Huang, et al., "Few-shot knowledge graph completion," arXiv preprint arXiv: 1911.11298, 2019.

[24] Z.-X. Ye and Z.-H. Ling, "Multi-level matching and aggregation network for few-shot relation classification," Annual Meeting of the Association for Computational Linguistics (ACL), pp.2872-2881, 2019.

[25] I. Balažević, C. Allen, and T.M. Hospedales, "TuckER: Tensor factorization for knowledge graph completion," arXiv preprint arXiv: 1901.09590, 2019.

[26] H. Sato, K. Otomo, and T. Masuo, "A knowledge sharing system using XML Linking Language and peer-to-peer technology," 2002 Symposium on Applications and the Internet, SAINT 2002 Proceedings, pp.26-27, 2002.

[27] J. Zhang, Y. Liu, and Y. Xiao, "Internet Knowledge-Sharing System Based on Object-Oriented," Second International Symposium on Intelligent Information Technology Application, vol.08, pp.239-243, 2008.

[28] J. Domingue, D. Fensel, and J. Hendle, "Introduction to the Semantic Web Technologies," Handbook of Semantic Web Technologies, Springer Berlin Heidelberg, pp.1-41, 2011.

[29] M.B. Raza and R. Harrison, "Ontological knowledge based system for product, process and resource relationships in automotive industry," CEUR Workshop Proceedings, vol.748, pp.23-36, 2011.

[30] U. Ghani, X. Zhai, J.M. Spector, N.-S. Chen, L. Lin, D. Ding, and M. Usman, "Knowledge hiding in higher education: role of interactional justice and professional commitment," Higher Education, vol.79, no.2, pp.325-344, 2020.

[31] I. Assouroko, G. Ducellier, P. Boutinaud, and B. Eynard, "Knowledge management and reuse in collaborative product development - a semantic relationship management-based approach," International Journal of Product Lifecycle Management, vol.7, no.1, pp.54-74, 2014.

[32] H. Jin, L. Hou, J. Li, and T. Dong, "Fine-Grained Entity Typing via Hierarchical Multi Graph Convolutional Networks," Proc. 2019 Conference on Empirical Methods in Natural Language Processing and the 9th International Joint Conference on Natural Language Processing (EMNLP-IJCNLP), pp.4969-4978, 2019.

[33] Z. Wang, K. Lai, P. Li, L. Bing, and W. Lam, "Tackling LongTailed Relations and Uncommon Entities in Knowledge Graph Completion," Proc. 2019 Conference on Empirical Methods in Natural Language Processing and the 9th International Joint Conference on Natural Language Processing (EMNLP-IJCNLP), pp.250-260, 2019.

[34] S. Xiao, Y.-J. Chen, and C.S. Tang, "Knowledge Sharing and Learning among Smallholders in Developing Economies: Implications,
Incentives, and Reward Mechanisms," Operations Research, vol.68, no.2, pp.435-452, 2020.

[35] D. Kimiz, "Knowledge Management in Theory and Practice," The MIT Press, 2011.

[36] Q. Cui, S. Wu, Q. Liu, W. Zhong, and L. Wang, "MV-RNN: A MultiView Recurrent Neural Network for Sequential Recommendation," IEEE Trans. Knowl. Data Eng., vol.32, no.2, pp.317-331, 2020.

[37] Y. Zhu, J. Lin, S. He, B. Wang, Z. Guan, H. Liu, and D. Cai, "Addressing the Item Cold-Start Problem by Attribute-Driven Active Learning," IEEE Trans. Knowl. Data Eng., vol.32, no.4, pp.631-644, 2020

[38] Y.J. Park, "The adaptive clustering method for the long tail problem of recommender systems," IEEE Educational Activities Department, 2013.

[39] J. Wang, "RH-Net: Improving neural relation extraction via reinforcement learning and hierarchical relational searching," arXiv preprint arXiv: 2010.14255 .

[40] P. Jeffrey, S. Richard, and M. Christopher, "GloVe: Global vectors for word representation," Proc. 2014 Conference on Empirical Methods in Natural Language Processing (EMNLP), pp.1532-1543, 2014.

[41] T. Wolf, L. Debut, and V. Sanh, "Transformers: State-of-the-art natural language processing," Proc. 2020 Conference on Empirical Methods in Natural Language Processing: System Demonstrations, pp.38-45, 2020.

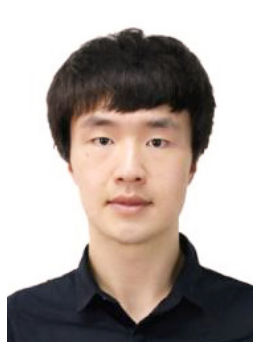

Yangshengyan Liu received the M.S. degree in Computer Science from the College of Computer Science \& Technology, Zhejiang University of Technology in 2019. During 20192020, he studied in the Department of Industrial and System Engineering, Zhejiang University. His research interests include lifecycle theory of industry, deep learning, and knowledge graph.

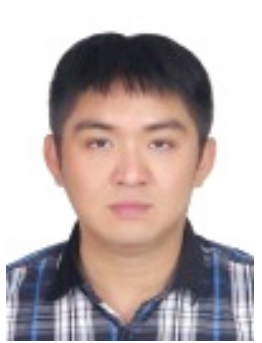

Fu Gu is an associate professor in Department of Industrial and System Engineering, and in Center of Engineering Management, Polytechnic Institute, Zhejiang University. $\mathrm{He}$ is a member of Chinese Society of Optimization, Overall Planning and Economic Mathematics.

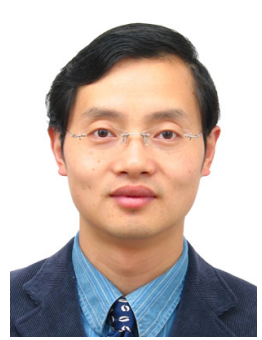

Yangjian Ji is currently a Professor of Zhejiang University and a deputy director of the Key Laboratory of Advanced Manufacturing Technology of Zhejiang Province. He received the Ph.D. degree in mechanical engineering from Zhejiang University in 2004. He is currently a member of Group Technology and Intelligent Integrated Technology of Chinese Mechanical Engineering Society. His research interests include manufacturing service, industrial big data, and advanced manufacturing mode. 


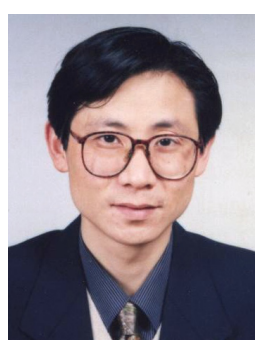

Yijie Wu is a Professor of School of Mechanical Engineering at Zhejiang University. In 1986, he received a Bachelor's Degree in Precision Machinery from Zhejiang University, and a Master's Degree in Mechanical Manufacturing from Zhejiang University in 1989 . He received his doctorate degree in mechanical manufacturing and automation from Zhejiang University in 1999 . Now, he has presided over one National High-tech R\&D Program of China (863), four National Natural Science Foundation Project and one Key Project of Zhejiang Provincial Natural Science Foundation.

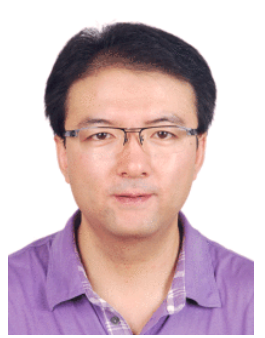

Jianfeng Guo is currently a professor at Institutes of Science and Development, Chinese Academy of Science, Beijing, China. He received his $\mathrm{Ph} . \mathrm{D}$. in engineering from Zhejiang University in 2007. His research interests include innovation theory, decision support systems, and environmental \& energy management. His works have appeared in journals including Nature Communications, Applied Economics, Physica A: Statistical Mechanics and Its Applications, Cluster Computing, Applied Energy.

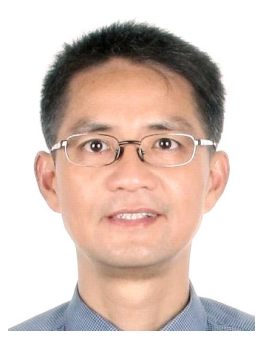

Xinjian Gu received Ph.D degree from Zhejiang University in 1993. He is the deputy director of research center for innovation management and sustained competition capability, Zhejiang University. His research interests include green manufacturing, manufacturing information engineering, advanced manufacturing technology, network manufacturing, mass customization, knowledge management, computer integrated manufacturing, group technology, mechanical manufacturing system and engineering, and industrial engineering.

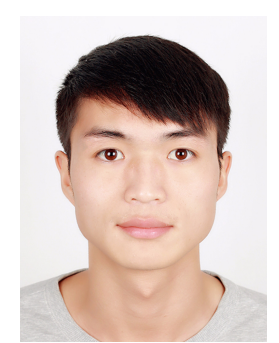

Jin Zhang received the B.E. degree in the Department of Industrial and System Engineering, Zhejiang University in 2017. He is currently studying for his $\mathrm{Ph} . \mathrm{D}$. degree at Zhejiang University. His research interests include artificial intelligence, knowledge management, and intelligent manufacturing. 